International Mathematical Forum, 1, 2006, no. 40, 1997-2002

\title{
SOME INFINITE CLASSES OF FULLERENE GRAPHS
}

\author{
Rashid Zaare-Nahandi \\ Institute for Advanced Studies in Basic Sciences \\ Zanjan, Iran
}

\begin{abstract}
A fullerene graph is a 3 regular planar simple finite graph with pentagon or hexagon faces. In these graphs the number of pentagon faces is 12. Therefore, any fullerene graph can be characterized by number of its hexagon faces. In this note, for any $h>1$, we will construct a fullerene graph with $h$ hexagon faces. Then, using the leapfrogging process we will construct stable fullerenes with $20+3 h$ hexagon faces, for any $h>1$.
\end{abstract}

\section{Mathematics Subject Classification: 92E10}

Keywords: Fullerene, cubic planer graph, leapfrogging process.

\section{Introduction}

A fullerene is a molecule consisting entirely of carbon atoms. Each carbon is three-connected to other carbon atoms by one double bond and two single bonds. These molecules are of great importance in chemistry: "Buckyball", one of famous fullerenes was named "Molecule of the Year" for 1991 by Science magazine. In the December 20, 1991, issue of Science, the Editors made the following observations: "Fullerene science exhibits the classic profile of a major scientific breakthrough. [6].

Since 1991, the pace of discovery in fullerene science has continued to accelerate. Researchers around the world are exploring both the basic science and potential applications of fullerenes. And the field has spawned important new areas of exploration, including carbon nanotubes and nanowires. In 1996, the Nobel Prize in Chemistry was awarded to the co-discoverers, Richard Smalley, Robert Curl and Harry Kroto, for their discovery of fullerenes. [9].

In this paper, we revisit the graph-theoretical formulation of fullerenes and their construction. A fullerene graph is a cubic planar graph with all 
faces 5-cycles or 6-cycles. Let the number of 5-cycles (pentagons) in a given fullerene $F$ is $p$ and number of 6 -cycles (hexagons) is $h$. Since each vertex lies in exactly 3 faces and each edge lies in 2 faces, then the number of vertices is $v=(5 p+6 h) / 3$, the number of edges is $e=(5 p+6 h) / 2=\frac{3}{2} v$, and the number of faces is $f=p+h$. By the Euler's formula $v-e+f=2$, one can deduce that

$$
\frac{5 p+6 h}{3}-\frac{5 p+6 h}{2}+p+h=2
$$

and therefore

$$
p=12, \quad v=2 h+20, \quad e=3 h+30 .
$$

Example 1. For $h=0$, the unique fullerene is dodecahedron with $v=$ $20, e=30$.

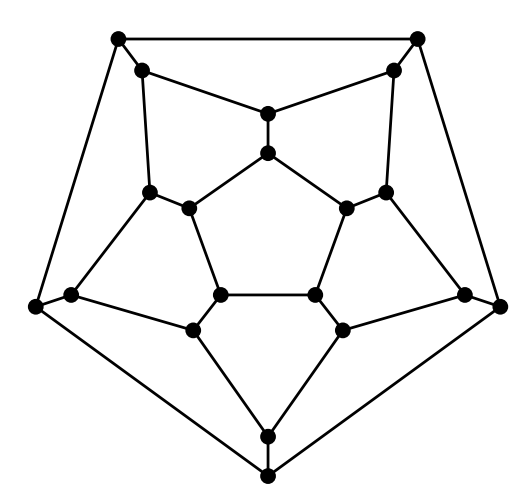

Figure 1: Fullerene $h=0$

\section{Constructing fullerenes}

A question can be posed: for a given natural number $h$, is there a fullerene graph with exactly $h$ hexagons? In this section we will see that for any $h>1$, there is at least one fullerene graph including exactly $h$ hexagons.

First step: constructing small fullerenes. In this step, we introduce six fullerene graphs with $2,3,4,5,6$ and 7 hexagons. For $h=0$ the fullerene graph 
is the above dodecahedron, and there is no fullerene graph with $h=1$.
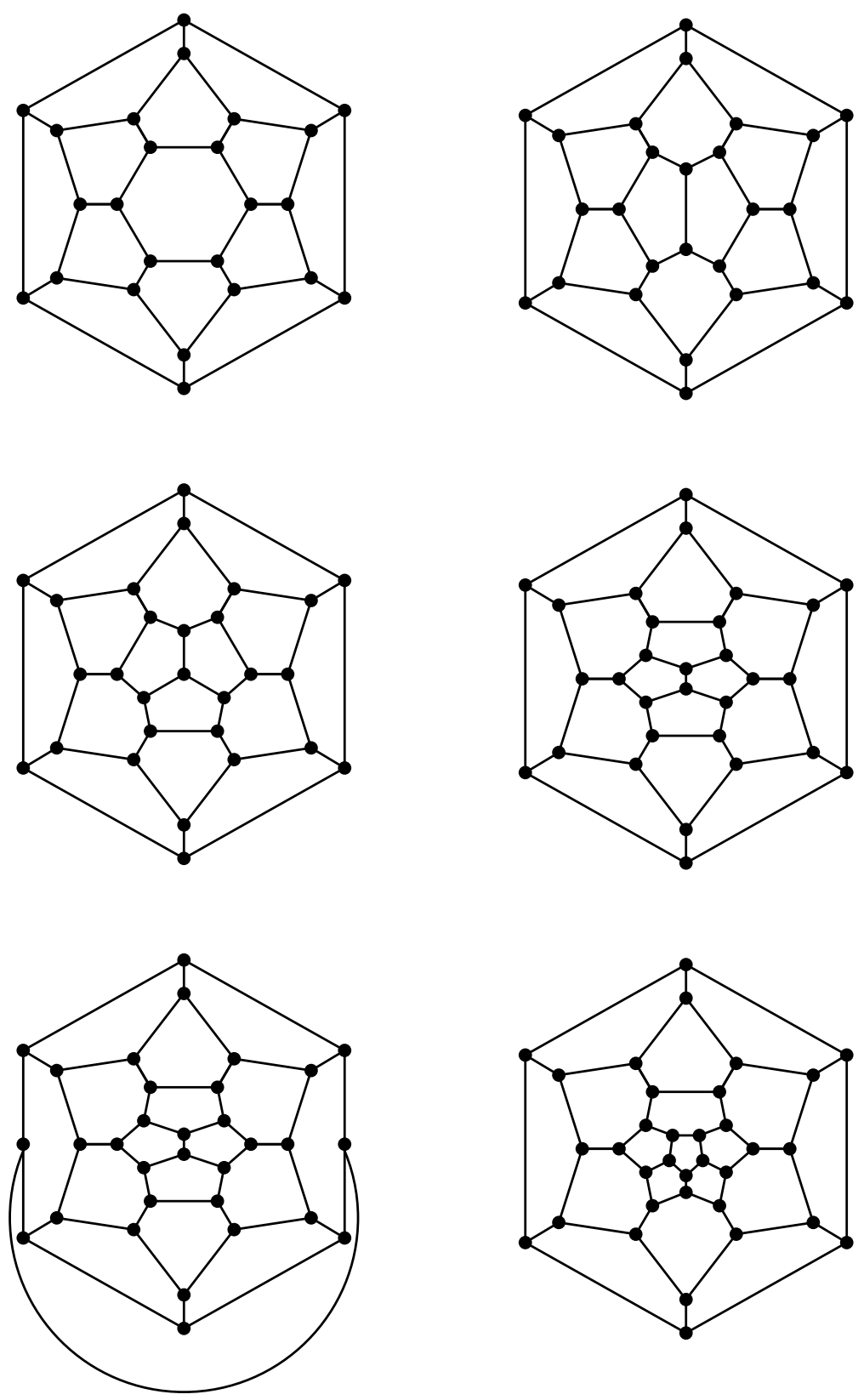

Figure 2: Fullerenes $h=2,3,4,5,6,7$.

In the above fullerenes, there is a common property (except in $h=6$ ): there is a hexagon (the outer one) which it's neighbors are all pentagons. Such fullerenes can be extended by an extending process in below. 
Extending process. Let $F$ be a fullerene with a hexagon face neighbored by pentagons only, as the above $h=2,3,4,5,7$. We may add a vertex to each edge of the hexagon to make all 6 neighboring pentagons, hexagon, and add an edge to each new vertex and finally join the ends of new edges to make a new hexagon. With this process, we get a new fullerene with 6 more hexagons. The new fullerene has the same property and we may do the process again to get new fullerenes. So, we can construct fullerenes with $h=i+6 k$ hexagons, for $i=2,3,4,5,7$ and $k=1,2,3, \ldots$. These numbers cover all natural numbers except multiples of 6 . But, the same process which is done for $h=5$ to get $h=6$ in Figure 2, can be done for any fullerene with $h=6 k-1$ hexagons to get a fullerene with $h=6 k$ hexagons.

Example 2. The extending process on $h=2$ to get $h=8$ is shown in Figure 3.
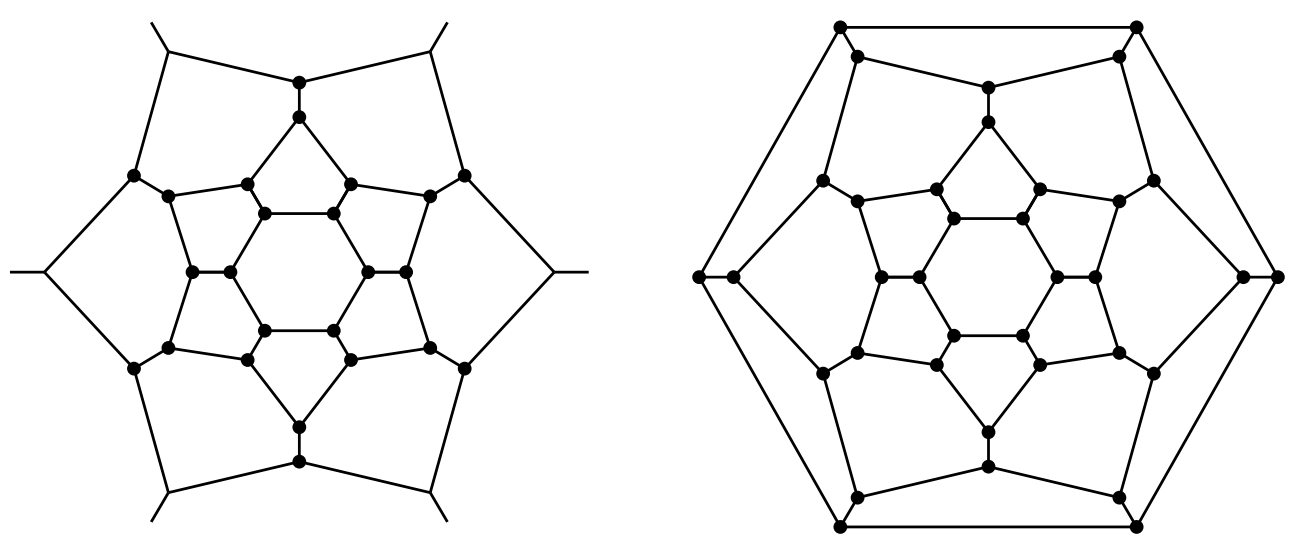

Figure 3: Extending $h=2$ to $h=8$

Theorem 2.1 For any natural number $h \neq 1$, there is a fullerene with exactly $h$ hexagons.

\section{Stable fullerenes}

There are some evidences that chemists believe that necessary conditions for physical existence of a particular fullerene are first that the pentagons must be isolated, that is, there are no two pentagons with a common edge; and second that the graph has exactly half of its eigenvalues positive and half negative. A fullerene with isolated pentagons is called isolated fullerene and an isolated fullerene with half eigenvalues positive and half negative is called stable fullerene. In this section, we will construct stable fullerenes by leapfrogging process. 
Leapfrogging process. [3] Let $F$ be a simple planar graph. Note that the outer cycle is also a face. We construct a new graph $L(F)$ whose vertices are $(e, f)$, where $e$ is an edge of $F$ and $f$ is a face of $F$ containing $e$. The vertices $\left(e_{1}, f_{1}\right)$ and $\left(e_{2}, f_{2}\right)$ are adjacent in $L(F)$ if one of the following conditions holds.

1) $e_{1}=e_{2}$ and $f_{1}$ and $f_{2}$ both contain $e_{1}$,

2) $f_{1}=f_{2}$ and $e_{1}$ has a common vertex with $e_{2}$ in $F$.

We say that $L(F)$ is obtained from $F$ by leapfrogging process. It is clear that if $F$ is a fullerene graph, then $L(P)$ is an isolated fullerene graph.

Example 3. By the leapfrogging process on the dodecahedron (Example 1), we get the Buckminsterfullerene.

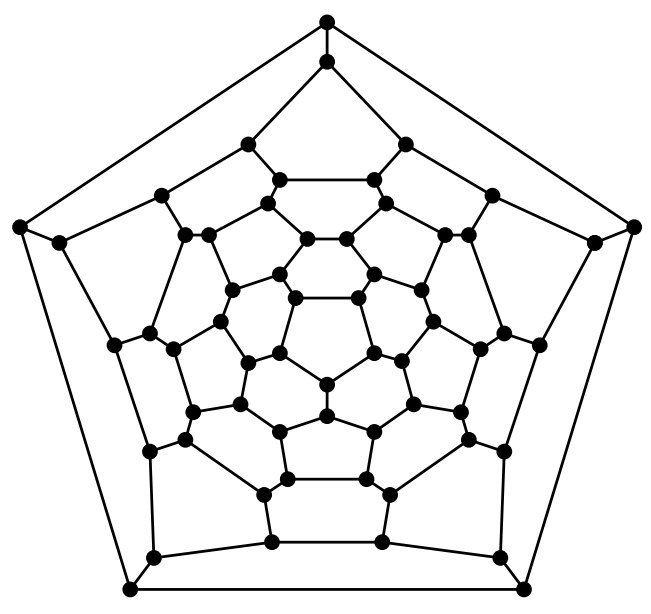

In other hand, by the following theorem, the fullerenes adopted by the leapfrogging process are stable.

Theorem 2.2 Let $F$ be a fullerene graph, then it's leapfrog graph $L(F)$ is stable. [3, Theorem 9.9.2]

If $F$ is a fullerene graph with $h$ hexagons, then it has $v=2 h+20$ vertices and $e=3 h+30$ edges. Therefore, $L(F)$ has $6 h+60$ vertices, $9 h+90$ edges and $3 h+20$ hexagons. We have proved following theorem.

Theorem 2.3 For any $h=0,2,3,4, \ldots$, there is a stable fullerene graph with exactly $3 h+20$ hexagons.

For instance, if $h=0$, we get the $C_{60}$ (Example 3), and for $h=2$ we adopt $C_{72}$, and etc.

ACKNOWLEDGEMENTS. This work is done with financial support of Institute for Advanced Studies in Basic Sciences. 


\section{References}

[1] T.W. Ebbesen and P.M. Ajayan, Large-scale synthesis of carbon nanotubes", Nature, 358 (1992), 220.

[2] S.H. Friedman, D.L. DeCamp, R.P. Sijbesma, G. Srdanov, F. Wudl and G.L. Kenyon, Inhibition of the HIV-1 Protease by Fullerene derivatives: model building studies and experimental verification, J. Am. Chem. Soc., 115 (1993), 6506.

[3] C. Godsil and G. Royle, Algebraic Graph Theory, Spriger-Verlag, GTM 207, New York, 2001.

[4] R.C. Haddon, L.E. Brus and K. Raghavachari, Electronic structure and bonding in icosahedral C, Chem. Phys. Lett., 125 (1986), 459.

[5] D.W. Iacoe, W.T. Potter and D. Teeters, Simple generation of C (Buckminsterfullerene), J. Chem. Ed., 69 (1992), 663.

[6] D.E. Koshland Jr. Buckyballs: wide open playing field for chemists", Science, 254 (1991), 1706.

[7] H.W. Kroto, J.R. Heath, S.C. O'Brien, R.F. Curl, and R.E. Smalley, C: Buckminsterfullerene, Nature, 318 (1985), 162.

[8] H.W. Kroto, The stability of the Fullerenes C, with $\mathrm{n}=24,28,32,36$, 50, 60, and 70, Nature, 329 (1987), 529.

[9] http://nobelprize.org 